\title{
Handwriting at Different Paces and Sizes With Visual Cues in Persons With Parkinson's Disease
}

\author{
Andrew F. Zaman a, b, Elizabeth L. Stegemoller ${ }^{\mathrm{a}}$
}

\begin{abstract}
Background: Persons with Parkinson's disease (PD) typically have small handwriting, especially when writing faster and/or larger. However, visual cues can help persons with PD increase their handwriting size. This study tested if lined paper would improve handwriting in persons with $\mathrm{PD}$, even when writing faster and/or larger. Secondarily, we wanted determine if persons with PD perceived handwriting as stressful, and if perceived stress was associated with writing performance.
\end{abstract}

Methods: The study included 22 subjects with Parkinson's disease and 11 age-gender-matched controls. Participants completed eight trials $(2 \times 2 \times 2)$ of printing a "P" and "d", at a comfortable speed and also as fast as possible in two different sizes $(1,2 \mathrm{~cm})$. The participants wrote with a ballpoint pen on lined paper. Bipolar electromyography (EMG) sensors recorded muscle activity from the index finger extensor (extensor digitorum communis (EDC)) and flexor (first dorsal interosseous (FDI)). Participants completed all of the trials for a particular pace (conditions were randomized) before completing all the trials of the other pace (order was counterbalanced).

Results: Handwriting height was smaller for persons with PD when required to write fast. There was also a trend for patients with PD to write slower and have smaller peak pen accelerations, but these were not statistically significant. Persons with PD found handwriting to be more stressful than healthy older adults did; and perceived stress negatively correlated with letter height and EMG activity.

Conclusions: Our study found that visual cues did not normalize handwriting height in persons with $\mathrm{PD}$ when writing large and/or fast. Persons with PD find handwriting to be stressful, and stress may negatively influence their handwriting.

Keywords: Parkinson's; Handwriting; Cue; Pace; Size; Stress

Manuscript submitted July 16, 2018, accepted August 27, 2018

aDepartment of Kinesiology, Iowa State University, Ames, IA 50011, USA ${ }^{b}$ Corresponding Author: Andrew F. Zaman, Department of Kinesiology, Iowa State University, 105 Barbara E. Forker Building, Ames, IA 50011, USA. Email: zaman@iastate.edu

doi: https://doi.org/10.14740/jnr493w

\section{Introduction}

Handwriting in persons with Parkinson's disease (PD) is smaller, slower, and often illegible [1-4]. These handwriting impairments become more differentiated when persons with PD are required to write larger and/or faster than normal [46]. External cues however, can help persons with PD improve their handwriting, gait, and other skills by directing their attention towards the task [7-9]. For example, persons with PD write larger if provided visual cues such as lined paper [10]. Others have also found improvements with visual cues, but that handwriting size was still smaller than that of healthy older adults (HOAs) when using tablets [11]. It remains unknown if persons with PD will write as large as HOAs when using visual cues on lined paper, or how their handwriting will be affected when required to write at larger than normal, or at a faster than normal pace. The main purpose of this study was to compare pen and paper handwriting with visual cues between persons with PD and HOAs at normal $(1 \mathrm{~cm})$ and a larger than normal size $(2 \mathrm{~cm})$, as well as at their self-preferred pace and a faster pace.

In healthy individuals, handwriting size is increased by generating greater forces and moving faster [12]. Persons with PD however, display abnormal force generation (e.g. decreased peak force amplitude, decreased rate of force production, greater latency of peak force production, and increased force variability) [13-15], which may contribute to small and slow handwriting $[4,16,17]$. For example, persons with PD demonstrate smaller peak pen tip accelerations and more inversions in acceleration patterns during handwriting $[4,5,18]$. While research has shown that the provision of visual cues can improve handwriting in PD, it remains unknown whether this improvement is due to improved force generation. Thus measuring changes in force generation (peak pen accelerations and muscle activity) across handwriting size and speed during visually cued handwriting in persons with PD may provide further insight into the role of force generation in handwriting.

A secondary purpose of this study is to examine the relationship between handwriting and stress. Because handwriting is often impaired and difficult for persons with PD, it may also be stressful. In healthy individuals stressful handwriting tasks result in increased force generation, movement speed, movement variation, and error rate $[19,20]$. Very few studies have examined how stress affects PD motor symptoms. Research has demonstrated that adding a stressful secondary task such as mental arithmetic during handwriting results in handwriting 

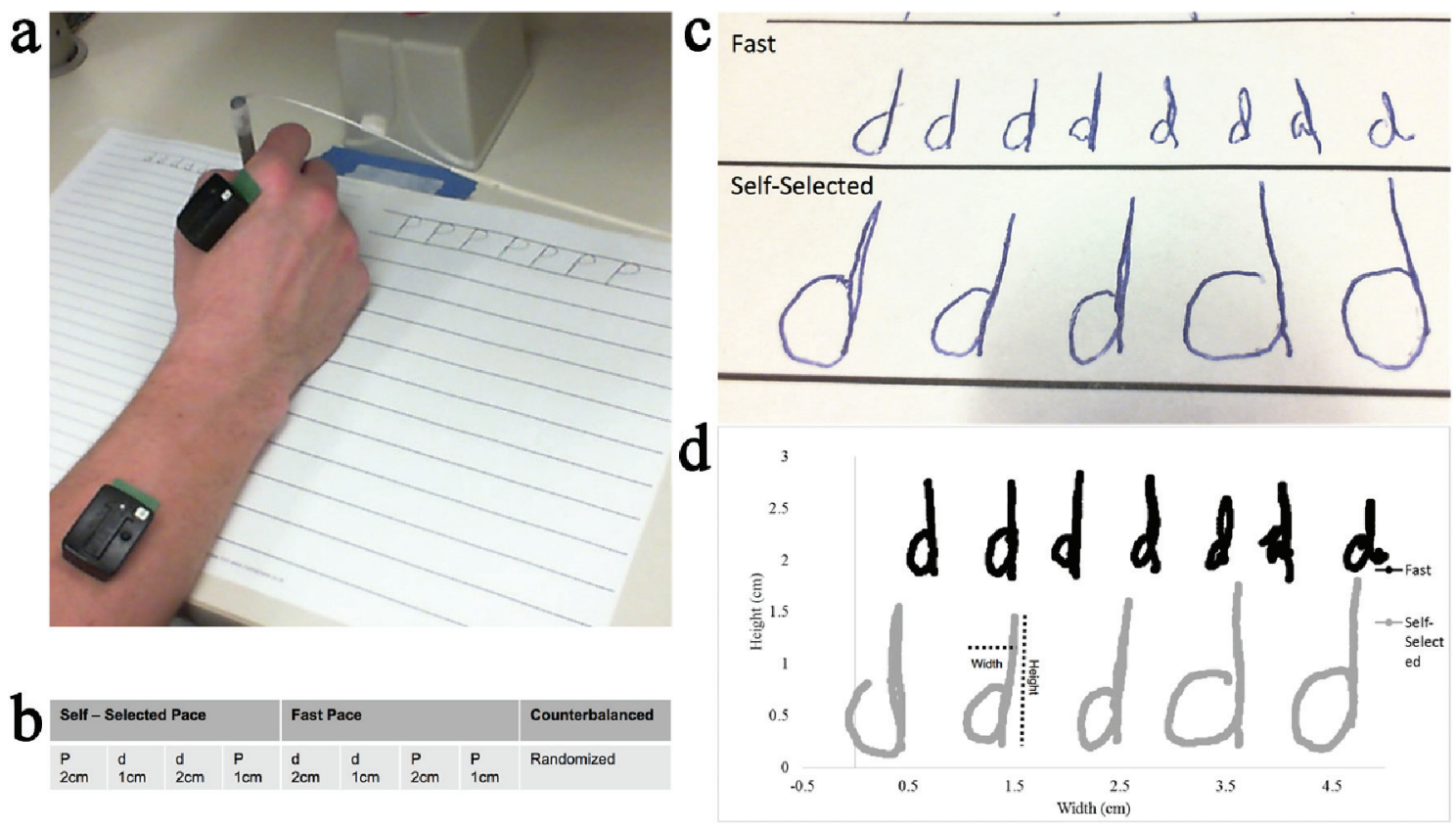

Figure 1. (a) Picture of experimental set up. (b) Experimental design. (c) Example handwriting at the $2 \mathrm{~cm}$ target size from a person with PD. The top row is the fast condition; the bottom row is the self-selected condition. (d) Example of the digital representation of handwriting.

performance decrements [5, 21]. It should be noted however, that the secondary tasks were cognitively demanding and that secondary tasks in general result in performance decrements $[22,23]$. Thus, this study will examine the relationship between perceived stress and handwriting in persons with PD, without the addition of a secondary task.

Given the aforementioned gaps in knowledge, this study will address the following hypotheses. Since visual cues can improve handwriting size in persons with $\mathrm{PD}$, we hypothesize that when using lined paper handwriting size, pen acceleration, and muscle activation would be improved in persons with PD regardless of the letter size or handwriting pace. However, given that stress can impair movement performance, we hypothesize that perceived stress would negatively correlate with handwriting size.

\section{Methods}

\section{Participants}

Twenty-two participants diagnosed with idiopathic PD (mean age $68.4 \pm 10.6$ years; eight males and 14 females), and 11 age-, gender-, and handedness-matched HOAs (mean age 70.7 \pm 9.5 years; two males and nine females) completed the handwriting tasks with their dominant hand. Participants with PD were recruited via the Iowa State University Neurophysiology Lab Parkinson's Disease Database, which consists of a list of individuals who have indicated an interest in being contacted about relevant research opportunities. Our participants with PD had Hohen and Yahr scores of between 1 and 3 (mean score
$2.0 \pm 0.6$ ), indicating mild to moderate $\mathrm{PD}$. The average number of years since diagnosis was $9.3 \pm 5.4$. Two persons with PD were left-handed. Participants were excluded if they had other neurological, cognitive, psychological, or musculoskeletal diseases that could confound results. Participants with PD were tested in their optimal medication state. All participants signed an informed consent. The informed consent was presented both verbally and via hard copy. This experiment was approved by the Iowa State University Institutional Review Board.

\section{Procedure}

A standard diameter ballpoint pen and two sheets of lined paper were provided. One sheet of paper had lines spaced $1 \mathrm{~cm}$ apart, and the other sheet had lines spaced $2 \mathrm{~cm}$ apart. Participants were instructed to write the letters "P" and " $\mathrm{d}$ " in print style on both sheets of paper, completely filling the space between the lines (Fig. 1a). The letters "P" and "d" were chosen because they contain both an upward or downward stroke and a circular curve to either the left or right. Participants wrote both letters at both sizes at both a fast pace and a self-selected pace for eight trials in a $2 \times 2 \times 2$ (letter $\times$ size $\times$ pace $)$ design. Each trial lasted $20 \mathrm{~s}$. The participants were instructed to write at the target size filling up the space between the top and bottom target lines. During the self-selected pace participants were instructed to write the letters at a "comfortable relaxed pace", and for the fast pace task they were instructed to "write as fast as possible". Participants completed all of the trials (letter and size) for a particular pace before completing all the trials of the other pace (order was counterbalanced between 
participants). The order in which target size and letter were written was randomized within each participant. An example of the order of trials is shown in Figure 1b.

Pen kinematics were recorded at $200 \mathrm{~Hz}$ from an electromagnetic sensor (Ascension), placed near the tip of the ballpoint pen. Bipolar electromyography (EMG) sensors (Delsys) were placed on the index finger extensor (extensor digitorum communis (EDC)), and flexor (first dorsal interosseus (FDI)) of the hand used to write (Fig. 1a). Perceived stress was recorded following each pace condition and was rated on a Likert scale of 1 (not at all stressful) to 10 (extremely stressful).

\section{Data analysis}

Kinematic outcome measures included letter height, letter width, letters completed, movement time, and acceleration. Muscle activity data were collected by examining peak EMG, and EMG area under the curve. To obtain the kinematic outcome measures, a digital representation of the handwriting was reproduced from the pen sensor data using a project-specific Java program (Fig. 1c, d). Periods of time when the pen was lifted off the page were filtered out so that the digital copy resembled the original handwriting. Letter height and width were calculated for each letter as the max excursion in the vertical and horizontal orientations of the page and averaged across each condition. Letters completed was the total number of complete letters for each trial. Instantaneous acceleration was calculated using the first central difference method [24]. Peak acceleration or the greatest instantaneous acceleration, was obtained for each letter and averaged for each condition.

To obtain the measures of muscle activation, the root mean square was calculated for the EMG signal from both muscles, and then was filtered with a 20 to $500 \mathrm{~Hz}$ band-pass filter and a $59-61 \mathrm{~Hz}$ notch filter. Peak EMG amplitude was calculated by averaging the peak EMG amplitude for each written letter, and was averaged for each condition. EMG area under the curve was calculated by adding the trapezoidal area between successive points for the duration of the trial.

In some instances, kinematic and EMG data were unable to be processed. In other cases, only one letter could be processed; and in those instances, the data from the single letter were used as the mean for the condition. For instances where no data could be processed, or data were missing for both letters, group mean substitution was used [25]. There was one instance each for letter width, peak pen acceleration, and EMG for the EDC where this occurred. The FDI EMG data had four participants with missing data, and due to the large percentage of missing data, pairwise deletion was used for those cases [26].

\section{Statistical analysis}

There appeared to be no differences in letter height, width, and speed between the two letters $(\mathrm{P}=0.95, \mathrm{P}=0.88$, and $\mathrm{P}=0.25$, respectively), so the two conditions were collapsed for further statistical analyses. To address the first hypothesis comparing handwriting between persons with $\mathrm{PD}$ and HOAs, a repeated measure analysis of variance (ANOVA) with a 2 (PD, control) $\times 2(1 \mathrm{~cm}$, and $2 \mathrm{~cm}$ target sizes) $\times 2$ (self-selected, and fast pace) factorial design was applied for each outcome measure (letter height, letter width, letters completed, movement time, peak acceleration, peak EMG, and EMG area under the curve). When interaction effects were revealed, $t$-test comparisons were completed with Bonferroni correction. For within-subject comparisons, two-tailed paired $t$-tests were completed. For between subjects' comparisons, a Levene's test was applied to determine equal or unequal variance before completing the subsequent two-tailed $t$-test.

To determine if participants found writing at a faster pace to be more stressful than writing at their self-selected pace, we used a two-way (group $\times$ pace) repeated measures ANOVA. Finally, the second hypothesis was addressed using a spearman partial correlation between perceived stress and all other outcome variables after controlling for group, size, and pace.

\section{Results}

Table 1 shows the means and standard deviation values for all outcome measures in both groups.

\section{Letter height}

Figure $2 \mathrm{a}$ shows the results for letter height. A significant main effect was revealed for group $(\mathrm{F}(1,31)=7.512, \mathrm{P}=0.01)$ and size $(\mathrm{F}(1,31)=394.409, \mathrm{P}<0.001)$, but not pace $(\mathrm{F}(1,31)=$ $4.116, \mathrm{P}=0.051)$. In general, participants with $\mathrm{PD}$ demonstrated smaller letter height compared to HOAs, and letter height was larger for the $2 \mathrm{~cm}$ size compared to the $1 \mathrm{~cm}$ size. A significant interaction effect was revealed for size $\times$ pace $(\mathrm{F}(1,31)$ $=20.812, \mathrm{P}<0.001)$, and pace $\times$ group $(\mathrm{F}(1,31)=4.456, \mathrm{P}=$ $0.043)$. No other interaction effects were revealed.

For the size $\times$ pace interaction effect, post hoc analysis revealed that across all participants, letter height was shorter in the fast condition for the $2 \mathrm{~cm}$ size $(-1.07 \pm 1.52 \mathrm{~mm}, \mathrm{t}(32)=$ $-4.047, \mathrm{P}<0.001)$, but not the $1 \mathrm{~cm}$ size $(0.04 \pm 0.88 \mathrm{~mm}, \mathrm{t}(32)$ $=0.276, \mathrm{P}=0.784)$. For the pace $\times$ group interaction effect, post hoc analysis revealed that across both sizes, persons with PD decreased letter height at the fast pace $(-0.78 \pm 1.48 \mathrm{~mm}$, $\mathrm{t}(43)=-3.499, \mathrm{P}=0.001)$, while HOAs did not $(0.02 \pm 0.87$ $\mathrm{mm}, \mathrm{t}(21)=0.083, \mathrm{P}=0.934)$.

\section{Letter width}

Figure $2 \mathrm{~b}$ shows the results for letter width. There was a significant main effect for group $(\mathrm{F}(1,31)=26.268, \mathrm{P}<0.001)$ and size $(\mathrm{F}(1,31)=159.666, \mathrm{P}<0.001)$, but not pace $(\mathrm{F}(1,31)$ $=0.236, P=0.63)$. In general, letters were significantly wider for the $2 \mathrm{~cm}$ target size compared to the $1 \mathrm{~cm}$ target size, and the participants with PD demonstrated significantly smaller letter width than HOAs. There were significant interaction effects for size $\times$ group $(F(1,31)=11.016, P=0.002)$ and pace $\times$ 
Table 1. Means and Standard Deviations for All Outcome Variables at Both Sizes and Both Paces

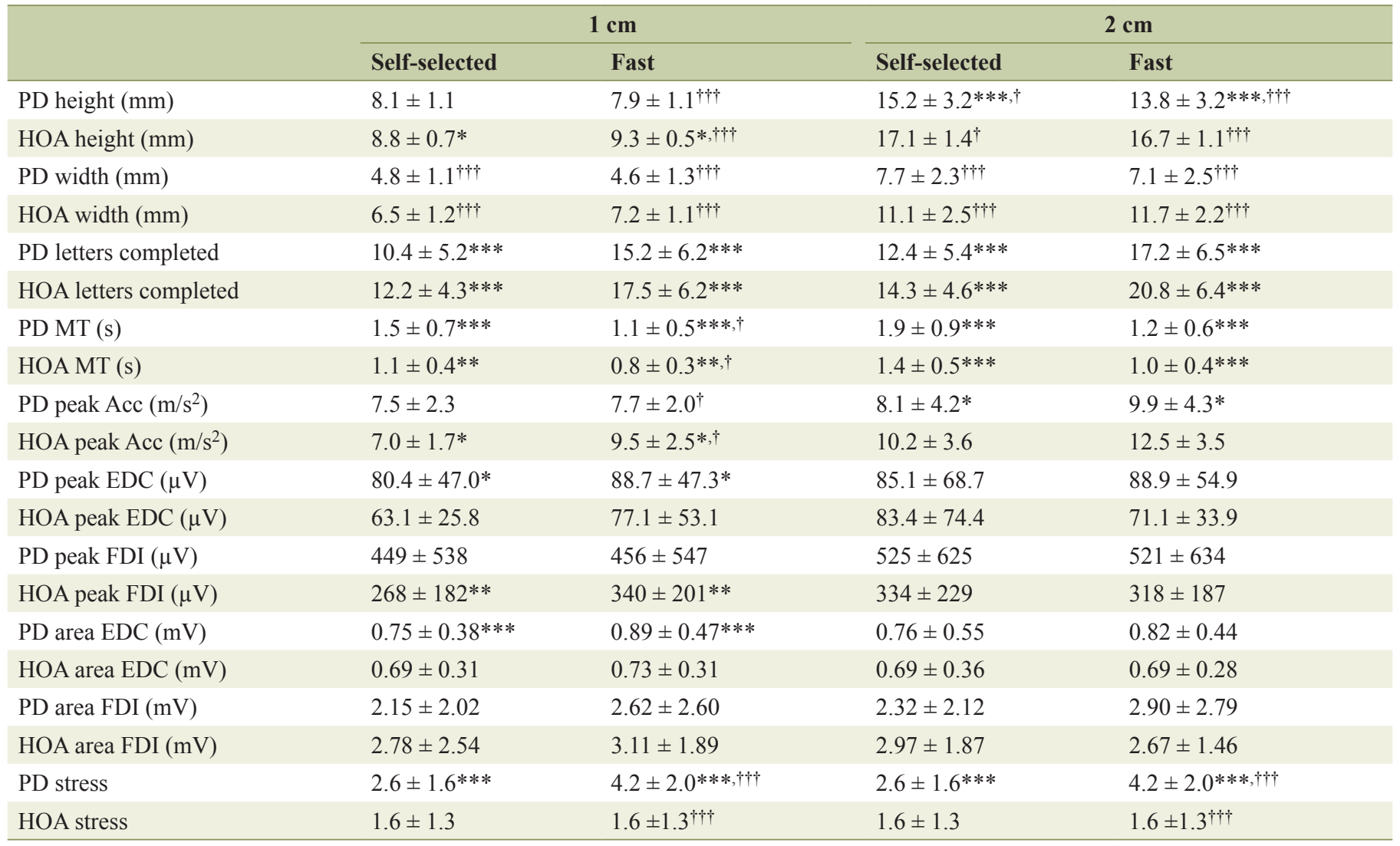

For within subjects pace comparisons at either size $(1 \mathrm{~cm}$ or $2 \mathrm{~cm})$, ${ }^{*} \mathrm{P}<0.05$, ${ }^{* *} \mathrm{P}<0.01,{ }^{* * *} \mathrm{P}<0.001$. For between subjects' comparisons for a particular size $\left(1 \mathrm{~cm}\right.$ or $2 \mathrm{~cm}$ ) and pace (self-selected or fast), ${ }^{\dagger} \mathrm{P}<0.05$, ${ }^{\dagger \dagger} \mathrm{P}<0.01$, ${ }^{\dagger \dagger} \mathrm{P}<0.001$. Acc: acceleration; EDC: extensor digitorum communis; FDI: first dorsal interosseus; HOAs: healthy older adults; PD: persons with Parkinson's disease; MT: movement time.

group $(\mathrm{F}(1,31)=7.188, \mathrm{P}=0.012)$. No other interaction effects were revealed.

For the size $\times$ group interaction effect, post hoc analysis revealed that both groups wrote wider letters at the larger size but that the difference was greater for HOAs (PD: $2.65 \pm 1.67$ $\mathrm{mm}, \mathrm{t}(43)=10.551, \mathrm{P}<0.0001 ; \mathrm{HOA}: 4.54 \pm 1.52 \mathrm{~mm}, \mathrm{t}(21)$ $=13.99, \mathrm{P}<0.0001)$. For the pace $\times$ group interaction effect, post hoc analysis revealed participants with PD decreased the letter width $(-0.44 \pm 1.10 \mathrm{~mm}, \mathrm{t}(43)=-2.644, \mathrm{P}=0.011)$ while HOA subjects increased their letter width $(+0.63 \pm 1.40 \mathrm{~mm}$, $\mathrm{t}(21)=2.107, \mathrm{P}=0.047)$ when writing faster.

\section{Letters completed, and movement time}

For letters completed and movement time, a significant main effect for pace $(\mathrm{F}(1,31)=65.698, \mathrm{P}<0.001 ; \mathrm{F}(1,31)=39.097$, $\mathrm{P}<0.001$ respectively) and size $(\mathrm{F}(1,31)=78.808, \mathrm{P}<0.001$; $\mathrm{F}(1,31)=27.535, \mathrm{P}<0.001$ respectively), but not group $(\mathrm{F}(1,31)=1.475, \mathrm{P}=0.234 ; \mathrm{F}(1,31)=2.609, \mathrm{P}=0.116$ respectively) were revealed. Participants completed more letters, and had shorter movement times when writing at the faster pace or when writing at the smaller $1 \mathrm{~cm}$ target size. No interaction effects were statistically significant for letters completed. For movement time, an interaction effect for size $\times$ pace $(\mathrm{F}(1,31)$ $=8.542, \mathrm{P}=0.006)$ was revealed. Participants wrote slower when writing larger, but the difference was greater during the self-selected pace $(0.31 \pm 0.32 \mathrm{~s}, \mathrm{t}(32)=5.697, \mathrm{P}<0.001)$ than the fast pace $(0.13 \pm 0.5 \mathrm{~s}, \mathrm{t}(32)=3.747, \mathrm{P}=0.001)$. No other interaction effects were revealed for movement time.

\section{Peak acceleration}

For peak acceleration there was a significant main effect for pace $(F(1,31)=10.560, P=0.003)$ and size $(F(1,31)=17.644$, $\mathrm{P}<0.0001)$, but not group $(\mathrm{F}(1,31)=2.883, \mathrm{P}=0.10)$. Participants had larger peak accelerations when writing at the faster pace and when writing larger. There was also a trend for the HOAs to have larger peak pen accelerations $(P=0.1)$. No interaction effects were statistically significant.

\section{Electromyography}

For peak EMG amplitude, no main or interaction effects were revealed for either the FDI or EDC. For FDI EMG area, a main effect for pace $(\mathrm{F}(1,27)=4.640, \mathrm{P}=0.040)$, but not for size 


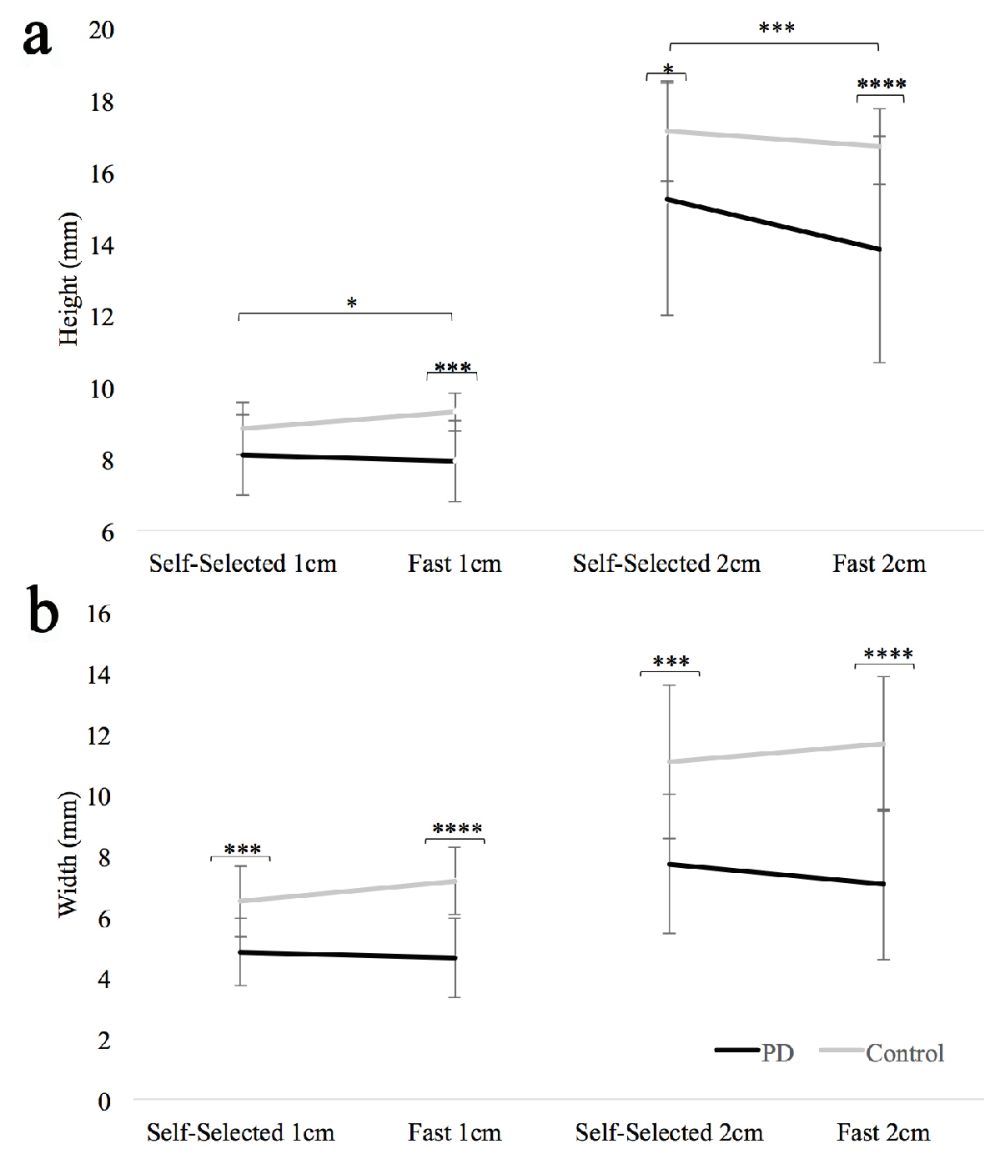

Figure 2. (a) Letter height for both groups at both paces (fast and self-selected) and both sizes $(1 \mathrm{~cm}$ and $2 \mathrm{~cm})$. (b) Letter width for both groups at both paces (fast and self-selected) and both sizes $(1 \mathrm{~cm}$ and $2 \mathrm{~cm}$ ). Standard error bars shown. Long horizontal bars show within group differences; the short horizontal bars show between group differences. ${ }^{*} \mathrm{P}<0.05$, ${ }^{* * *} \mathrm{P}<0.001$, ${ }^{* * * *} \mathrm{P}<$ 0.0001 .

$(\mathrm{F}(1,27)=1.603, \mathrm{P}=0.216)$, or group $(\mathrm{F}(1,27)=0.003), \mathrm{P}=$ $0.956)$ was revealed. In general, participants had greater FDI EMG area when writing at the fast pace, and at the larger target size. No main effects were found for EDC EMG area, and no interaction effects were found for either the FDI or EDC EMG area.

\section{Perceived stress}

Figure 3a shows results for perceived stress. There was a main effect of group $(\mathrm{F}(1,31)=10.847, \mathrm{P}=0.002)$, and pace $(\mathrm{F}(1,31)=7.494, \mathrm{P}=0.01)$. In general, participants found writing at a fast pace to be more stressful, and persons with PD found handwriting to be more stressful than HOAs. There was also a pace $\times$ group interaction $(\mathrm{F}(1,31)=7.494, \mathrm{P}=0.01)$. Post hoc analysis revealed that persons with PD found it more stressful $(\mathrm{P}<0.001)$ to write at a fast pace compared to a selfselected pace, while control subjects did not $(\mathrm{P}=1.0)$. Post hoc analysis also showed that persons with $\mathrm{PD}$ found writing at a faster pace to be more stressful than HOAs $(\mathrm{P}<0.001)$, while the perceived stress between the groups at a self-selected pace was statistically comparable $(\mathrm{P}=0.79)$. Scatter plots of perceived stress and letter height, perceived stress and peak FDI EMG, and perceived stress and FDI EMG are shown in Figure $3 \mathrm{~b}$, Figure 3c, and $3 \mathrm{~d}$ respectively. After correcting for pace, size and group, stress was significantly correlated with height $(P=0.036)$, peak FDI EMG $(P=0.007)$, and FDI EMG area $(P=0.009)$ (Table 2). Stress did not correlate with any of the remaining outcome measures.

\section{Discussion}

Our results revealed that when using lined paper, participants with PD and HOAs displayed statistically comparable handwriting height during the $1 \mathrm{~cm}$, self-selected pace condition. In comparison, Nackaerts and colleagues (2016) found that when using a tablet, persons with PD still had smaller handwriting at a $1 \mathrm{~cm}$ size despite a visual cue mediated increase in size [11]. Thus, there may be some differences in the effectiveness of visual cueing when using paper as compared to a tablet. Another possibility is that there were differences in the visual cues used. Nackerts and colleagues used a $2 \mathrm{~mm}$ target zone 

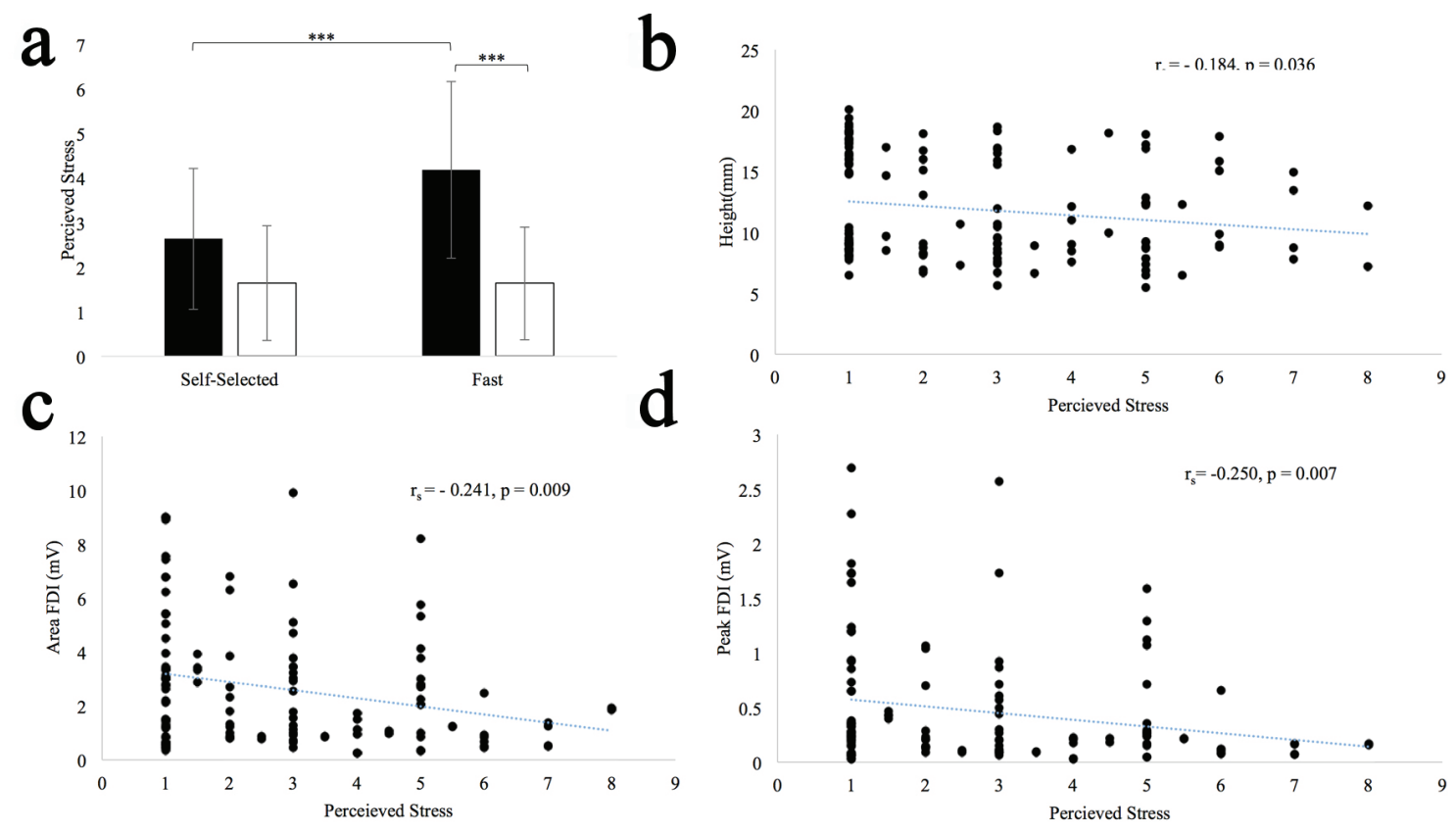

Figure 3. (a) Difference between perceived stress for self-selected and fast pace conditions. Standard error bars shown. Long horizontal bars show within group differences; the short horizontal bars show between group differences. ${ }^{* * *} P<0.001$. (b) Perceived stress vs. height scatter plot. (c) Perceived stress vs. peak FDI EMG scatter plot. (d) Perceived stress vs. FDI EMG scatter plot.

instead of a much smaller target line, or another possibility is that the letters used (cursive lower case "l" instead of the letters "P" and "d") also had an effect on the handwriting size [11]. For all other conditions, persons with PD had shorter letters than HOAs. These results demonstrated that lined paper was unsuccessful in normalizing handwriting size when participants with PD were required to write fast and/or large despite using pen and paper.

Previous research has demonstrated that in persons with PD lined paper does not improve word width [21, 27]. In our

Table 2. Spearman Partial Correlation Between Perceived Stress and All Other Outcome Variables After Controlling for Group, Size, and Pace

\begin{tabular}{lll}
\hline & \multicolumn{2}{c}{ Perceived stress } \\
\cline { 2 - 3 } & $\mathbf{r}_{\mathbf{s}}$ & $\mathbf{P}$ \\
\hline Height $(\mathrm{mm})$ & $-0.184^{*}$ & 0.036 \\
Width $(\mathrm{mm})$ & 0.059 & 0.504 \\
Letters completed & 0.113 & 0.203 \\
MT $(\mathrm{s})$ & -0.095 & 0.285 \\
Peak Acc $\left(\mathrm{m} / \mathrm{s}^{2}\right)$ & 0.132 & 0.137 \\
Peak EDC $(\mu \mathrm{V})$ & 0.049 & 0.579 \\
Peak FDI $(\mu \mathrm{V})$ & $-.250^{* *}$ & 0.007 \\
Area EDC $(\mathrm{mV})$ & 0.075 & 0.4 \\
Area FDI $(\mathrm{mV})$ & $-0.241^{* *}$ & 0.009 \\
\hline
\end{tabular}

${ }^{*} \mathrm{P}<0.05 .{ }^{* *} \mathrm{P}<0.01$. ACC: acceleration; EDC: extensor digitorum communis; FDI: first dorsal interosseus; MT: movement time. study, persons with PD also wrote letters that were smaller in width during all of the conditions. Interestingly though, persons with PD decreased their letter width during the fast writing conditions, whereas controls increased letter width. Taken together, the use of cues to improve handwriting seems only to apply to small, self-paced handwriting. When handwriting is more challenging, visual cues are not as effective. These results also suggest that cues may be pertinent to the domain of improvement (e.g. height, width) in persons with PD.

When writing at a self-selected pace, persons with $\mathrm{PD}$ typically write slower than HOAs [3, 4]. However, it has been shown that when asked to write fast, persons with PD can write just as quickly as HOAs $[17,18]$. When using lined paper, persons with PD write slower [10, 21, 27]. In our study, persons with PD wrote at statistically similar albeit slower pace (movement time, letters competed), suggesting that both HOAs and persons with PD modified the speed at which they wrote in a similar manner. One possibility is that participants in this study may have focused more on speed and less on size, and given their force production impairments, they were unable to modulate their height without slowing down.

The peak pen accelerations of both groups were very similar during the $1 \mathrm{~cm}$ self-selected pace. There was however, a trend $(\mathrm{P}=0.1)$ for persons with $\mathrm{PD}$ to have smaller peak pen accelerations. Post hoc comparisons showed that while peak pen accelerations were smaller in persons with $\mathrm{PD}$, they were statistically similar for all but the $1 \mathrm{~cm}$ fast-paced condition. In healthy individuals, handwriting size is modulated by increasing force production, which is estimated by looking at peak pen accelerations $[4,12,18]$. Force production is abnormal in persons with $\mathrm{PD}$, and is a potential mechanism underlying 
their slow and small handwriting [16]. For example, studies have shown that persons with PD have smaller peak pen accelerations, more sub-movements per stroke, and an inability to scale pen accelerations when writing at larger sizes $[3,4$, 16]. Despite not reaching statistical significance, it appears that persons with PD did not increase their force production enough to ameliorate differences in handwriting size during the large and/or fast conditions. In cases of writing faster and/ or larger persons with PD would have to slow down and produce longer stroke durations to see an improvement in size.

Something unique to our study is the measurement of muscle activity in the flexors (FDI) and extensors (EDC) of index finger. The index finger is involved in gripping the pen and the act of writing. We found no group differences in overall activation of the FDI and EDC. However, the overall FDI activation scaled with handwriting speed, that is the FDI became more active during the fast pace conditions. This was surprising, as persons with PD typically have reduced force production. More testing is needed however to determine the group differences in index finger muscle recruitment during handwriting. For the EDC, we found no main or interaction effects for either overall or peak activation, which suggests that the EDC is minimally involved in handwriting.

In general, persons with PD perceived handwriting to be more stressful than HOAs. They also found writing at a fast pace to be more stressful than writing at a comfortable pace. This is the first study to demonstrate that even a simple and common task, like handwriting, can be stressful for persons with PD. Results also revealed that perceived stress negatively correlated with FDI muscle activation (peak and overall) and letter height. Wilson (2008) suggested that stress is distracting, and that stress negatively effects attention and one's ability to utilize visual cues [28]. As previously mentioned, cues are thought to be effective by increasing task-directed attention $[7,8]$. Consequently, stress could negatively affect, or at least negate the positive effects of external cues. Indeed, results revealed that stress is negatively correlated with letter height even though a visual cue was present. Results also demonstrated that lower levels of FDI muscle activation (peak and overall) were found in the more stressed individuals. This could be an artifact of handwriting speed. However, for persons with PD, peak FDI means were nearly identical in both the fast and self-paced conditions, but they still reported an increase in perceived stress. Thus, the results of this study suggest that increasing the speed of handwriting may cause additional stress, and negatively impact force production and handwriting size.

\section{Limitations}

One limitation of this study is that we did not compare handwriting with visual cues (lined paper) with non-cued handwriting. We can only determine if PD handwriting with visual cues is similar to that of HOAs. Another limitation is that we presented cues for both pace (write "as fast as you can", or at a "comfortable pace") and size (parallel lines), making it hard to tell which cue was more pertinent to the participant. However, we feel that the results are a good representation of the natural writing conditions, as individuals may be pressured to write large and/or fast. Stress was only measured following the fast pace conditions and the slow pace conditions. Thus, we did not measure stress when writing at large sizes compared to normal sizes, it is likely that writing larger is more stressful than writing at a normal size as well. Finally, caution should be used when interpreting the role of stress and its effect on PD motor symptoms. In the case of our study, it could be those with more impaired handwriting found it to be more stressful, and that stress had no direct impact on handwriting. This study may also have a selection bias, as our participants with PD were individuals who indicated an interest in being contacted about research opportunities.

\section{Conclusions}

Persons with PD have impaired performance in routine tasks such as handwriting. Overall, our study found that visual cues did not normalize pen and paper handwriting height in persons with PD when writing large and/or fast. Results also demonstrated that persons with PD find handwriting to be more stressful than HOAs. Thus, increasing the speed and height of writing may cause additional stress, which negates the benefit of a visual cue in persons with PD. Future research is needed to determine if stress similarly influences other simple tasks so that more effective therapeutic interventions, including decreasing stress, can be developed for persons with PD.

\section{Declaration}

None.

\section{Funding}

This study was supported by Iowa State University.

\section{References}

1. Letanneux A, Danna J, Velay JL, Viallet F, Pinto S. From micrographia to Parkinson's disease dysgraphia. Mov Disord. 2014;29(12):1467-1475.

2. Smits EJ, Tolonen AJ, Cluitmans L, van Gils M, Conway BA, Zietsma RC, Leenders KL, et al. Standardized handwriting to assess bradykinesia, micrographia and tremor in Parkinson's disease. PLoS One. 2014;9(5):e97614.

3. Tucha O, Mecklinger L, Thome J, Reiter A, Alders GL, Sartor H, Naumann M, et al. Kinematic analysis of dopaminergic effects on skilled handwriting movements in Parkinson's disease. J Neural Transm (Vienna). 2006;113(5):609-623.

4. Van Gemmert AW, Adler CH, Stelmach GE. Parkinson's disease patients undershoot target size in handwriting and similar tasks. J Neurol Neurosurg Psychiatry. 2003;74(11):1502-1508. 
5. Broeder S, Nackaerts E, Nieuwboer A, Smits-Engelsman BC, Swinnen SP, Heremans E. The effects of dual tasking on handwriting in patients with Parkinson's disease. Neuroscience. 2014;263:193-202.

6. Van Gemmert AW, Teulings HL, Stelmach GE. Parkinsonian patients reduce their stroke size with increased processing demands. Brain Cogn. 2001;47(3):504-512.

7. Morris ME, Iansek R, Summers JJ, Matyas TA. Motor control considerations for the rehabilitation of gait in Parkinson's disease. Adv Psychol. 1995;111:61-93.

8. Morris ME, Iansek R. Characteristics of motor disturbance in Parkinson's disease and strategies for movement rehabilitation. Human Movement Science. 1996;15(5):649669.

9. Morris ME, Iansek R, Matyas TA, Summers JJ. Stride length regulation in Parkinson's disease. Normalization strategies and underlying mechanisms. Brain. 1996;119 (Pt 2):551-568.

10. Oliveira RM, Gurd JM, Nixon P, Marshall JC, Passingham RE. Micrographia in Parkinson's disease: the effect of providing external cues. J Neurol Neurosurg Psychiatry. 1997;63(4):429-433.

11. Nackaerts E, Nieuwboer A, Broeder S, Smits-Engelsman BC, Swinnen SP, Vandenberghe W, Heremans E. Opposite effects of visual cueing during writing-like movements of different amplitudes in Parkinson's disease. Neurorehabil Neural Repair. 2016;30(5):431-439.

12. Thomassen AJ, Teulings HL. Time, size and shape in handwriting: Exploring spatio-temporal relationships at different levels. In Time, mind, and behavior, Springer Berlin Heidelberg. 1985; pp. 253-263.

13. Neely KA, Planetta PJ, Prodoehl J, Corcos DM, Comella CL, Goetz CG, Shannon KL, et al. Force control deficits in individuals with Parkinson's disease, multiple systems atrophy, and progressive supranuclear palsy. PLoS One. 2013;8(3):e58403.

14. Stelmach GE, Teasdale N, Phillips J, Worringham CJ. Force production characteristics in Parkinson's disease. Exp Brain Res. 1989;76(1):165-172.

15. Wascher E, Verleger R, Vieregge P, Jaskowski P, Koch S, Kompf D. Responses to cued signals in Parkinson's disease. Distinguishing between disorders of cognition and of activation. Brain. 1997;120 (Pt 8):1355-1375.
16. Phillips JG, Martin KE, Bradshaw JL, Iansek R. Could bradykinesia in Parkinson's disease simply be compensation? J Neurol. 1994;241(7):439-447.

17. Van Gemmert AW, Teulings HL, Contreras-Vidal JL, Stelmach GE. Parkinson's disease and the control of size and speed in handwriting. Neuropsychologia. 1999;37(6):685-694.

18. Teulings HL, Stelmach GE. Control of stroke size, peak acceleration, and stroke duration in Parkinsonian handwriting. Human Movement Science. 1991;10(2):315334.

19. van Galen GP, van Huygevoort M. Error, stress and the role of neuromotor noise in space oriented behaviour. Biol Psychol. 2000;51(2-3):151-171.

20. Van Gemmert AW, Van Galen GP. Stress, neuromotor noise, and human performance: a theoretical perspective. J Exp Psychol Hum Percept Perform. 1997;23(5):12991313.

21. van Gemmert AW, Teulings HL, Stelmach GE. The influence of mental and motor load on handwriting movements in parkinsonian patients. Acta Psychol (Amst). 1998;100(1-2):161-175.

22. Hackney ME, Earhart GM. The effects of a secondary task on forward and backward walking in Parkinson's disease. Neurorehabil Neural Repair. 2010;24(1):97-106.

23. O'Shea S, Morris ME, Iansek R. Dual task interference during gait in people with Parkinson disease: effects of motor versus cognitive secondary tasks. Phys Ther. 2002;82(9):888-897.

24. Wood GA. Data smoothing and differentiation procedures in biomechanics. Exerc Sport Sci Rev. 1982;10:308-362.

25. Allison PD. Missing data: Sage University Papers Series on quantitative applications in the social sciences, 07136. Thousand Oaks, CA, 2001.

26. Graham JW. Missing data analysis: making it work in the real world. Annu Rev Psychol. 2009;60:549-576.

27. Raudmann M, Taba P, Medijainen K.Handwriting speed and size in individuals with Parkinson's disease compared to healthy controls: the possible effect of cueing. Acta Kinesiologiae Universitatis Tartuensis, 2014;20:40-47.

28. Wilson M. From processing efficiency to attentional control: a mechanistic account of the anxiety-performance relationship. Int Rev Sport Exer P. 2008;1(2):184-201. 